EDUCATIONAL

-RESOURCE

Volume 11 Issue 12019

DOI: 10.21315/eimj2019.11.1.7

ARTICLE INFO

Submitted: 28-12-2018

Accepted: 03-02-2019

Online: 29-03-2019

\title{
Palliative Care for Disease with Uncertain Disease Trajectory: An Ethical Case Learning
}

\author{
Fahisham Taib ${ }^{1}$ Eisha Fatima², Jamil Abdullahi Faruk², Syed Abdul \\ Khaliq Syed Abdul Hamid ${ }^{4}$ \\ ${ }^{1}$ Paediatric Department, Hospital Universiti Sains Malaysia, \\ Kelantan, MALAYSIA \\ ${ }^{2}$ Paediatrics Department, University Hospital of Wales, Cardiff, \\ UNITED KINGDOM \\ ${ }^{3}$ Paediatrics Department, Faculty of Medicine, Ahmadu Bello \\ University, Zaria, Kaduna, NIGERIA \\ ${ }^{4}$ Paediatrics Department, International Islamic University Malaysia, \\ Kuantan Campus, Pahang, MALAYSIA
}

To cite this article: Taib F, Fatima E, Faruk JA, Syed Abdul Hamid SAK. Palliative care for disease with uncertain disease trajectory: an ethical case learning. Education in Medicine Journal. 2019;11(1):53-57. https://doi.org/10.21315/eimj2019.11.1.7

To link to this article: https://doi.org/10.21315/eimj2019.11.1.7

\section{ABSTRACT}

Disease trajectory in children can be difficult to determine. This is primarily, despite the life limiting condition, healthcare professionals are unable to pin the exact time when these children's lives will end. We illustrate an unusual case of dicephalic parapagus, an inseparable conjoined twin which was complicated by anomalies, psychosocial and safeguarding issues. Such case posed a challenge even to paediatric palliative care team as the trajectory of the disease, in this case, is rather difficult to predict. Anticipatory symptoms management perhaps is the best way forward, despite having to face many ethical challenges and medical enigma.

Keywords: Palliative care, Conjoined twin, Disease trajectory, Psychosocial

\section{INTRODUCTION}

Conjoined twins are extremely rare phenomenon. It occurs in 1 in 50,000 to 100,000 live births. For dicephalic parapagus, it always derives from a single fertilised ovum with monochorionic and monoamniotic features. Anatomically, the twin has fused or two hearts, two sets of lungs and commonly shared liver, gallbladder, pancreas, genitourinary and gastrointestinal tract. Risk of structural anomalies such as neural tube defect, imperforate anus and other organs are high
(1). It is difficult to predict a life span for twin with such complex medical needs.

\section{CASE SUMMARY}

Eight-month-old twin girls were transferred from foreign country under a charity sponsorship for corrective heart surgery. After extensive assessment, they were concluded to be inseparable due to multiple major organ anomalies. The twins had a normal heart in Twin 1 and complex congenital heart disease in Twin 2; a 
shared liver and guts; two gallbladders and hepatic venous systems; three kidneys with hydroureter and hydronephrosis and a single bladder. Twin 1 maintains an oxygen saturation of $100 \%$ while Twin 2 has saturation over $70 \%$. Each twin has a separated motor control over half of the body. Propranolol was commenced with the underlying cardiac anomaly. The twins had small bowel obstruction, which was surgically corrected by forming a colostomy. Both were on prophylactic antibiotic to prevent urinary tract infection. Socially, there has been a long history of domestic violence where the twin's father suffered significant physical abuse from the mother. Since then, the mother has abandoned the family. Father has applied for right to asylum following this episode. Palliative care team was involved for symptom management following complicated medical and social background.

\section{ISSUES}

The principle of palliation in children is rather different from their adult counterparts. Palliative care in children usually starts early, with a proactive approach and totality of care. Together for Short Lives stated that; Palliative care for children and young people is an active and total approach to care, from the point of diagnosis or recognition, throughout the child's life, death and beyond. It embraces physical, emotional, social and spiritual elements and focuses on the enhancement of quality of life for the child or young person and support for the family. It includes the management of distressing symptoms, provision of short breaks, care at the end of life and bereavement support (2).

The primary challenge was related to the difficulty on predicting the disease trajectory. It requires collaboration of multi-professional teams at the point of the diagnosis, crisis points along the natural course of the condition, with the involvement of family members in the difficult decision-making and resolving tough ethical issues. Parents often expect curative options rather than listening to sad news on death and dying. Palliative care in children requires a proactive approach to symptoms care and management. The twins are likely to have a steady decline of health due to inoperable cyanotic heart condition in of them. This would eventually limit their survival time. Their survival at the current state is due to compensatory cardiovascular mechanism from normal twin, despite having a different physiological structure compared to a normal human body. Prediction on the overall future outcome of this condition may well be difficult to determine (3).

The second challenge was related to drugs utilisation in this condition. Most of the drugs given in paediatrics depend on surface area or weight of the patients. In an obese child, the safest option would be to average the weight using a percentile chart of that specific age. Drug use in a conjoint twin can be tricky. Do we treat the twins as "one" or "two" different bodies? Estimating ideal body weight for each of them, for drug administration such as antibiotics, would be difficult. There was dilemma in choosing normal or separate weight calculation, bearing in mind that the twins were physiologically, anatomically and biochemically different but connected to each other. The easiest way is to take the whole weight and treat them as "one" being. Such approach may be inadequate to cover drugs dosage for two persons. Similarly, if one is to use estimated "two" human model, there is a likelihood to overzealously treating for both of them. For example, is it necessary to treat a healthy twin diuretic to control heart failure in the sicker twin? Should we be individualising our treatment approach? As the twins have different anatomical functioning organs, it would be important to consider the physiology for the compensatory mechanism and functioning for each of them. A shared liver and gut implied that oral medications would be metabolised in a same system. Declining of organs' function, such as heart and kidney, may point towards overall disease limitation. 
It is a conundrum for any physician when treating them; and if the approach would benefit nor harming the patients, this would be an acceptable path.

This equation of weight-age for medical intervention would not stop here. It would be influenced by many other decision processes. For example, vaccination in the future may be hampered with many issues. Although the twins can be referred as two individuals, but due their physical attachment, management should be tailored accordingly. The twins may be subjected to polypharmacy and sometimes unnecessary medications. Some palliative care drugs are unlicensed for children, mistakes may lead to excess dosage and drug error (4). Palliative care team prefers traditionally effective drugs to target different symptoms modality. For example, gabapentin is mainly used to control seizure, but it also has the property to combat visceral hyperalgesia and neuropathic pain.

There was also identified complex psychosocial issues. It is essential to keep the twin's development in a comfortable, normal and conducive environment. They were brought over specifically for medical management by an oversea charity group. We were uncertain as to how the fund was obtained. The twins' mother had physically assaulted the father and had left him alone to care for the children which has led to child protection discussion, whilst the father seek for asylum (5). Safeguarding needs should prompt a careful examined to avoid the risk of manipulation and victimisation. The right of the children is to be loved and developed according to family values, preferences and wishes.

Another vital issue was pertaining to the suitability of Paediatric Advanced Care (PAC) planning discussion (6). This is essential due to uncertainty of the twins' disease trajectory. There are various emotional issues surrounding decision to release the information, the timing and appropriateness of the PAC plan discussion. PAC plan is not a binding rule, but it would be helpful to have it completed to avoid mismanagement during the emergency scenario. This is not a "Do Not Resuscitate" order and can be changed loosely by the parents.

\section{ETHICAL JUSTIFICATION}

Clinical ethics analysis using four topics model is used to provide clinicians with a framework for sorting through and focusing on specific aspects of clinical ethics cases and for connecting the circumstances of a case to their underlying ethical principles (7). First, issues related to medical indication such as goal of treatment, prognostication or good death were explored. Many of non-oncological conditions in paediatric would be hard to predict on the future outcome and disease trajectory. Communication on unwelcomed news that their children's short life span can be devastating news for the father. Surgical separation of the twin may be detrimental for both due to high risk and complex surgical procedures. This route would go against the principle of beneficence and non-maleficence. Standard decision should be based on "best interest" model. Family may claim that the best interest of the child is based on parental dominant role and knowing their own children's best interest. Most parents are deeply committed to their child's wellbeing and will bear the consequences of their decision making. In certain circumstances, emotional influences may cloud their judgement. Some families made the decision based on estimated suffering; perhaps voicing the outcry by other family members. The deliberative model aims to aid the physician-patient interaction and to help the patient/parents determine and choose the best health related values that can be realised in that clinical situation (8).

Predicting the twins' prognosis may lead to inaccuracies and miscalculation. Optimal management would depend on the twins' needs at different stage of life and the ability to give family the informed choices (3). 
Justification for appropriate treatment goals is important to avoid unnecessary escalation of emergency care. It would require a lengthy discussion with the family members, parallel planning ahead and coordination of care around various teams involved.

Underage children's decision is largely influenced "by proxy", from parental or legal caregivers' judgement. Wishes and hopes should be articulated to reach mutual decision-making and extent to understanding the information. The twins' autonomy is limited by factors such as age, family request and capacity to understand or give consent. If there is a conflict of preferences between the family and doctors, negotiation should be allowed for an appropriate joint decision. Decision-making for a single parent, as depicted in this case, would be difficult due to complexity of the condition and contentious issues such as palliating the twin. The father would be changing normal fatherhood's role to full time caregiving. Healthcare physicians need to allow father to fathom available information on medical technicalities and allowing father to make a moral and conscientious decision based on his preferences.

Quality of life is a subjective reference towards individual experienced in life. Good quality of life would require different strategies for different conditions and individuals. Parent or patients may not have clear goal setting due to indifference of the disease trajectory, internal conflict and medical crises. Palliative care is often understood as a method to prolonging suffering, hastening death by having interventions that could lead to unnecessary duration of life. These could complicate matters when surrogate decision is made on behalf of dependent children. Discussion on the difficult matter during the crisis, such as the cardiopulmonary resuscitation discussion, may upset family members but this is necessary to allow sufficient time for understanding the topics discussed. Future planning on support, proposed end of life care and parallel planning need to be addressed in a sympathetic way. Although the subjects raised would cause anxiety and uncertainties, but this would give parent information and the chance to anticipate the outcome of this condition. Resolving the psychosocial and spiritual dimension would allow parent to have a control in their life and preparing for the worst-case scenario.

In the contextual discussion, drugs use in palliative care should be based on patients' symptomatology and its indication. Some children will require multiple drugs to achieve symptom control, and this indirectly would avoid distressing the family members. Communication is an essential tool to relay information and explanation on a specific medical decision. Drugs which are unlicensed for the children may be used, however, careful rationalisation of the medications needed to ensure proper indication and avoiding unwanted drug error and side effect. Off-label drug is medication that are prescribed outside their licensed indications with respect to dosage, age, indication, or route, and sometimes the formulation is modified as extemporaneous preparations (9). Physicians must always question whether the steps taken to use medications are justifiable, without potential harm and consequences. Parallel to this, medical management should be managed on case by case basis. The best interest of child should be a priority when considering any medical decision process.

Safeguarding is a complex ethical dilemma issue. Domestic violence is regularly associated with child abuse. The child protection protocol has not been activated once the mother of the twin abandoned them by travelling back to her country of origin. There are questions about the ethics of using public fund from other country which was speculated to bear the cost of the twin operation. Here are many moral justifications and dilemma - is it right for the father to obtain financial aid for palliative mean and sustenance in a foreign country whilst the money raised to cover the cost of surgery, is it right for father to obtain asylum entry based on his twins' demise, is 
there any risk of future domestic violence should the family return home? These are lingering questions that would be difficult to answer. PAC plan which was a non-binding agreement between the managing team and the parents for appropriate approach for end of life care, is used to simplify medical approach. It is not an "absolute" legal paper; many of these steps can be changed according to parental wishes. However, in the context of medical management, PAC plan would be crucial to rationalisation of approach during the end of life care.

\section{CONCLUSION}

Managing twin with uncertain prognostication and disease trajectory can be very challenging. There were series of medical approach, social dogma and ethical dilemma behind this case. There is no straightforward way out when managing complex children with chronic illnesses. The use of medical indication, parental preferences, exploring quality of life and contextual issues analysis may solve some of the ethical difficulties. There is no right and wrong approach to ethical analysis; but the basic tenets such as beneficence, nonmaleficence, justice, and finally respect for children and parent's autonomy would be a vital step towards a successful resolution of any ethical context.

\section{REFERENCES}

1. Harma M, Harma M, Mil Z, Oksuzler C. Vaginal delivery of dicephalic parapagus conjoined twin: case report and literature review. Tohoku J Exp Med. 2005;205:17985. https://doi.org/10.1620/tjem.205.179

2. Bergstraesser E. Pediatric palliative care: a reflection on terminology. Palliat Care. 2013;7:31-6. https://doi.org/10.4137/PCRT. S12800

3. Brook L, Hain R. Predicting death in children. Arch Dis Child. 2008;93:1067-70. https://doi.org/10.1136/adc.2007.127332
4. Ivanovska V, Rademaker CMA, van Dijk L, Mantel-Teeuwisse AK. Pediatric drug formulations: a review of challenges and progress. Pediatrics. 2014;134:361-72. https://doi.org/10.1542/peds.2013-3225

5. Newbigging K, Thomas N. Good practice in social care for refugee and asylum seeking children. Child Abuse Rev. 2011;20:374-90. https://doi.org/10.1002/car.1178

6. Fraser J, Harris N, Berringer AJ, Prescott $H$, Finlay F. Advanced care planning in children with life-limiting conditions the Wishes Document. Arch Dis Child. 2010;95:79-82. https://doi.org/10.1136/adc. 2009.160051

7. Schumann JH, Alfandre D. Clinical ethical decision making: the four topics approach. Semin Med Pract. 2008;11:36-42.

8. Hardart MKM, Truog RD. Spinal muscular atrophy-type I. Arch Dis Child. 2003;88:848-50. https://doi.org/10.1136/adc. 88.10 .848

9. Pandolfini C, Bonati M. A literature review on off-label drug use in children. Eur J Pediatr. 2005;164:552-8. https://doi.org/10. 1007/s00431-005-1698-8 